OPEN ACCESS

Edited by:

Stephen Tobias Abedon,

The Ohio State University,

United States

Reviewed by:

Katarzyna Małgorzata

Danis-Włodarczyk,

The Ohio State University,

United States

Rodolfo García-Contreras, Universidad Nacional Autónoma

de México, Mexico

*Correspondence:

Fuquan Hu

hufuquan2009@aliyun.com

Shuguang LU

shulang88@126.com

Specialty section:

This article was submitted to Antimicrobials, Resistance

and Chemotherapy,

a section of the journa

Frontiers in Microbiology

Received: 18 January 2018 Accepted: 15 May 2018

Published: 01 June 2018

Citation:

Li G, Shen M, Yang Y, Le S, Li M, Wang J, Zhao Y, Tan Y, Hu Fand

Lu S (2018) Adaptation of Pseudomonas aeruginosa to Phage PaP1 Predation via O-Antigen Polymerase Mutation.

Front. Microbiol. 9:1170. doi: 10.3389/fmicb.2018.01170

\section{Adaptation of Pseudomonas aeruginosa to Phage PaP1 Predation via O-Antigen Polymerase Mutation}

\author{
Gang Li, Mengyu Shen, Yuhui Yang, Shuai Le, Ming Li, Jing Wang, Yan Zhao, Yinling Tan, \\ Fuquan $\mathrm{Hu}^{*}$ and Shuguang $\mathrm{Lu}$ *
}

Department of Microbiology, Army Medical University, Chongqing, China

Adaptation of bacteria to phage predation poses a major obstacle for phage therapy. Bacteria adopt multiple mechanisms, such as inhibition of phage adsorption and CRISPR/Cas systems, to resist phage infection. Here, a phage-resistant mutant of Pseudomonas aeruginosa strain PA1 under the infection of lytic phage PaP1 was selected for further study. The PaP1-resistant variant, termed PA1RG, showed decreased adsorption to $\mathrm{PaP} 1$ and was devoid of long chain $\mathrm{O}$-antigen on its cell envelope. Whole genome sequencing and comparative analysis revealed a single nucleotide mutation in the gene PA1S_08510, which encodes the O-antigen polymerase Wzy that is involved in lipopolysaccharide (LPS) biosynthesis. PA1_Wzy was classified into the O6 serotype based on sequence homology analysis and adopts a transmembrane topology similar to that seem with $P$. aeruginosa strain PAO1. Complementation of gene wzy in trans enabled the mutant PA1RG to produce the normal LPS pattern with long chain O-antigen and restored the susceptibility of PA1RG to phage PaP1 infection. While wzy mutation did not affect bacterial growth, mutant PA1RG exhibited decreased biofilm production, suggesting a fitness cost of PA1 associated with resistance of phage PaP1 predation. This study uncovered the mechanism responsible for PA1RG resistance to phage PaP1 via wzy mutation and revealed the role of phages in regulating bacterial behavior.

Keywords: phage therapy, phage resistance, adsorption inhibition, Pseudomonas aeruginosa, O-antigen polymerase, biofilm

\section{INTRODUCTION}

Bacteriophages (phages) are viruses that can infect and kill the bacterial hosts specifically. Interaction between phages and their bacterial hosts is important in molecular biology research and has been extensively studied for decades (Chaturongakul and Ounjai, 2014). In various environments, phages and bacteria work toward into an endless state of co-evolutionary competition and equilibrium (De Smet et al., 2017). On the one hand, the high abundance of phages, which outnumber bacteria by approximately 10 -fold, makes the encounter of bacteria and phage invaders possible in every ecosystem (Sharma et al., 2017). To survive and/or escape phage predation, bacteria have evolved and acquired sets of resistance mechanisms, including prevention of phage adsorption to cell surfaces and subsequent injection of phage genomes, targeted cleavage of injected nucleic acids via restriction-modification (R-M) system and CRISPR/Cas system, and even suicide of phage-infected cells via abortive-infection (Abi) system (Labrie et al., 2010; 
Samson et al., 2013). On the other hand, despite the presence of these arsenals, phages have also adapted to overcome bacterial defense systems through several counter-strategies (Samson et al., 2013), such as mutation in specific phage genes (Pepin et al., 2008; Michel et al., 2010), phage genome rearrangement, and exchange with other viral or bacterial sequences (Labrie and Moineau, 2007). The constant competition and co-evolution contribute greatly to the genetic diversity of both bacteria and phages on the biosphere (Chaturongakul and Ounjai, 2014).

Pseudomonas aeruginosa is a Gram-negative opportunistic pathogen that causes various infections mainly in immunocompromised individuals, especially for those suffering from burn wounds, cancer and cystic fibrosis (Stover et al., 2000; Turner et al., 2015). The notorious characteristics of metabolic versatility, biofilm formation, and drug resistance make $P$. aeruginosa-related infections very difficult to eradicate in clinical settings (Mathee et al., 2008; Valot et al., 2015). Phages represent a promising alternative to traditional antibiotics for treating bacterial infections (Nobrega et al., 2015), particularly for multidrug-resistant and biofilm infections (Nobrega et al., 2015; Chan et al., 2016a). Despite the impressive specificity and efficiency of phage therapy, phage resistance still poses a major obstacle to its broad application (Nilsson, 2014). Therefore, a comprehensive understanding of the interactions between phages and bacteria, as well as phage resistance mechanisms, is still urgently required to predict and limit potentially undesired outcomes during phage-based applications.

Pseudomonas aeruginosa strain PA1 was originally isolated from a patient suffering from a respiratory tract infection and resists treatment by multiple antibiotics (Lu et al., 2015; Li et al., 2016b). Recent work has shown that upon predation by the lytic phage PaP1, PA1 produced phage-resistant variants at a frequency of $3 \times 10^{-5}$, and these variants could be classified into two major phenotypes: one produces red pigment homogentisic acid (Red mutant), whereas the other displays blue-green pigment pyocyanin (Green mutant) (Le et al., 2014). The features of phage resistance and pigment changes could be stably maintained for these mutants, suggesting potential genetic mutations within the PA1 genome. Comparative genomic analysis revealed a $219.6 \mathrm{~kb}$ genomic fragment deletion in the Red mutant (termed PA1r), and this deleted DNA fragment containing the key gene $h m g A$ and galU. Loss of $h m g A$ contributes to the accumulation of a red compound, homogentisic acid, whereas galU deletion results in hindering of bacterial lipopolysaccharide (LPS) biosynthesis, which functions as the receptor for phage PaP1 adsorption (Le et al., 2013). By contrast, the Green mutant produces the same pigment (pyocyanin) as the parental strain PA1. However, the underlying molecular basis for the phage $\mathrm{PaP} 1$ resistance of the Green mutant remains unknown.

In this study, a Green mutant of $P$. aeruginosa PA1 was characterized and termed as PA1RG. Comparative genomic analysis combined with molecular characterization revealed the crucial role of $\mathrm{O}$-antigen polymerase mutation in conferring the phage PaP1 resistance to PA1RG. The results of this work enhances our understanding of phage resistance mechanisms of bacteria.

\section{MATERIALS AND METHODS}

\section{Bacteria and Growth Conditions}

Pseudomonas aeruginosa strains PA1, PA1RG, and PA1RG/wzy were cultured in $\mathrm{LB}$ broth at $37^{\circ} \mathrm{C}$ unless otherwise specified. When necessary, $100 \mu \mathrm{g} / \mathrm{ml}$ gentamicin was supplemented for PA1RG/wzy culture. The value of $\mathrm{OD}_{600}$ for both PA1 and PA1RG cultures was measured every $30 \mathrm{~min}$ to profile the growth curve. The production of green pigment pyocyanin was photographed at the early-stationary phase.

\section{Adsorption Assay}

Phage PaP1 attachment ability was determined as previously described with minor modifications (Le et al., 2014). Briefly, log-phase culture of bacteria was washed and resuspended with LB broth to $10^{8} \mathrm{cfu} / \mathrm{ml}$. Phage PaP1 was then added to the bacterial suspension at a final concentration of $10^{5} \mathrm{pfu} / \mathrm{ml}$ and was incubated at $37^{\circ} \mathrm{C}$ for $5 \mathrm{~min}$. The samples were filtered, and phage titer in the supernatants was quantified. Adsorption ability was represented as [(total titer - titer in the supernatant)/total titer] $\times 100 \%$.

\section{LPS Profiling}

Lipopolysaccharide was isolated using the hot water-phenol method as described previously (Yi et al., 2009). Purified LPS was subjected to $12 \%$ SDS-PAGE and visualized by silver staining as formerly described (Fomsgaard et al., 1990).

\section{SMRT Sequencing of PA1RG Genome}

Complete genome sequence of PA1RG was determined on the PacBio RSII instrument (Pacific Biosciences, Menlo Park, $\mathrm{CA}$, United States). Libraries of $5-\mathrm{kb}$ were constructed for PA1RG genomic DNA, and four SMRT cells of the libraries were sequenced with 90-min movies. De novo assembly using RS_HGAP_Assembly version 2.0 (Chin et al., 2013), revealed a single contig of $6,500,439 \mathrm{bp}$ in length with 320 -fold sequence coverage (Li et al., 2016a).

The genome sequences of strain PA1, PA1RG, and PA1r are available at GenBank under the accession number CP004054, CP012679, and CP004055, respectively.

\section{Bioinformatics Analysis}

Sequence homology was searched using BLAST service at the NCBI website ${ }^{1}$. Multiple alignment of Wzy was performed by Clustal W (Larkin et al., 2007), and visualized using ESPript (Robert and Gouet, 2014). Wzy homologs used for phylogenetic analysis were retrieved from literature (Islam and Lam, 2014; Pan et al., 2016). Phylogenetic tree was constructed by MEGA using the neighbor-joining method (Saitou and Nei, 1987; Tamura et al., 2013). The transmembrane topology of Wzy was analyzed by software TMHMM 2.0 (Krogh et al., 2001).

\footnotetext{
${ }^{1}$ http://www.ncbi.nlm.nih.gov/BLAST
} 


\section{Construction of the Complementary Strain PA1RG/wzy}

The intact gene $w z y$ as well as its promoter region was amplified from PA1 genome using forward primer $5^{\prime}$ GGGGTACCCTTGCCGTCACTTTCTCCGA-3' (KpnI site underlined) and reverse primer 5'-AACTGCAGGGAGT TGGCGCATATGCATA-3' (PstI site underlined). The obtained PCR fragment was cloned into the KpnI/PstI sites of plasmid pUCP24 (West et al., 1994), resulting in pUCPwzy. Construct pUCPwzy was then electroporated into PA1RG to obtain the complementary strain PA1RG/wzy as described previously (Li et al., 2017).

\section{Spot Assay}

Sensitivity of PA1, PA1RG, and PA1RG/wzy to phage PaP1 was determined as described previously (Bondy-Denomy et al., 2016). Briefly, bacteria were firstly cultured to log-phase, and $200 \mu \mathrm{l}$ of aliquot mixed with $3 \mathrm{ml}$ of soft $\mathrm{LB}$ agar $(0.7 \%$, w/v) was poured onto the LB agar plate $(1.5 \%, \mathrm{w} / \mathrm{v})$ and solidified at room temperature. The phage $\mathrm{PaP} 1$ lysate $\left(10^{10} \mathrm{pfu} / \mathrm{ml}\right)$ was serially diluted in 10-fold steps, and $3 \mu$ l of dilutions was applied to the lawn of each strain, and bacterial lysis was assessed.

\section{Pyocyanin Quantitation Assay}

Pyocyanin production was quantitated as previously described (Essar et al., 1990). Briefly, a $5 \mathrm{ml}$ of culture supernatant grown in LB broth was extracted with $3 \mathrm{ml}$ of chloroform. After centrifugation, the chloroform layer was re-extracted into $1 \mathrm{ml}$ of $0.2 \mathrm{~N} \mathrm{HCl}$ to obtain a pink to deep red solution. The absorbance of this solution was quantified at $520 \mathrm{~nm}$.

\section{Microtiter Dish Biofilm Formation Assay}

The microtiter dish biofilm formation assay was performed as previously described (O'Toole, 2011). Briefly, overnight culture of PA1, PA1RG, and PA1RG/wzy was diluted 1:100 into fresh LB medium, respectively. Then, $100 \mu \mathrm{l}$ of aliquot was inoculated into the flat-bottom 96-well dish (Corning, United States) with six replicate wells for each strain. Biofilm was formed at $37^{\circ} \mathrm{C}$ for $24 \mathrm{~h}$. After incubation, the plate was washed with $\mathrm{ddH}_{2} \mathrm{O}$ to remove unattached cells and media components, followed by staining with $125 \mu \mathrm{l}$ of $0.1 \%$ crystal violet (Sangon Biotech, Shanghai, China). The plate was washed three times with $\mathrm{ddH}_{2} \mathrm{O}$ again and dried at room temperature. $125 \mu \mathrm{l}$ of $30 \%$ acetic acid (Sangon Biotech, Shanghai, China) was added to solubilize the crystal violet, and then transferred to a new flat-bottom microtiter dish. The absorbance was quantified in a plate reader at $550 \mathrm{~nm}$. Three biological repeats were performed for biofilm formation assay.

\section{Confocal Laser Scanning Microscopy (CLSM)}

Biofilm formation examined by CLSM was performed as described previously with minor modifications (Takenaka et al., 2001). Briefly, overnight culture of PA1, PA1RG, and PA1RG/wzy was diluted 1:100 into fresh LB medium, respectively. Then, $2 \mathrm{ml}$ of aliquot was inoculated into the glass-bottom cell culture dish
(15 $\mathrm{mm}$ in diameter, Nest, China), and incubated at $37^{\circ} \mathrm{C}$ for $24 \mathrm{~h}$. The biofilm was washed with phosphate-buffered saline (PBS), and fixed with $2.5 \%$ glutaraldehyde for $1.5 \mathrm{~h}$. After washing with PBS, the biofilm was labeled by $5 \mu \mathrm{M}$ fluorescent nucleic acid stain SYTO 61 (Invitrogen, United States) at room temperature for $30 \mathrm{~min}$, followed by $50 \mu \mathrm{g} / \mathrm{ml}$ FITC-labeled concanavalin A (FITC-ConA, Sigma-Aldrich, United States) at $37^{\circ} \mathrm{C}$ for $5 \mathrm{~min}$. The biofilm was visualized using the confocal laser scanning microscope LSM800 (Zeiss, Germany) with $561 \mathrm{~nm}$ excitation and $640 \mathrm{~nm}$ emission wavelengths for SYTO 61, and 488 and $537 \mathrm{~nm}$ for FITC-ConA, respectively.

\section{Statistical Analysis}

Data were processed using GraphPad Prism (GraphPad Software Inc., San Diego, CA, United States). When necessary, Student's $t$-test was applied, and the difference was considered statistically significant at $p<0.05$.

\section{RESULTS}

\section{PaP1 Infection of $P$. aeruginosa Resulted in Phage-Resistant Mutant PA1RG}

When the early log-phase culture of $P$. aeruginosa strain PA1 $\left(10^{8}\right.$ cfu) was infected with phage PaP1 $\left(10^{10} \mathrm{pfu}\right)$ at an MOI of 100 , the cloudy bacterial fluid became transparent, seemingly all the bacteria were killed by phage PaP1 (Figure 1A). However, several surviving bacteria grew colonies when plating the transparent lysate on an LB agar plate. This finding suggested that the phage-resistant mutants were selected by PaP1. Approximately two-thirds of the surviving bacteria grew colonies with no pigment produced on the screening plate, and one of these colonies was randomly selected for future analysis. Actually, the selected phage-resistant mutant could produce blue-green pigment in liquid media, which is identical to that of wild-type PA1 (Figure 1A). Thus it was named as PA1RG (Resistant Green mutant). No significant difference was identified for pyocyanin production between PA1 and PA1RG (Figure 1B), indicating that the biosynthesis of pigment pyocyanin was not impaired in mutant PA1RG. Phage PaP1 could efficiently lyse PA1 but not PA1RG even at high phage titer, confirming the phage-resistant phenotype of PA1RG (Figure 1C).

\section{Whole Genome Single-Molecule Real-Time (SMRT) Sequencing of PA1RG}

To probe the possible genetic or epigenetic variations within the PA1RG genome that conferred the phage-resistant phenotype, the PA1RG genomic DNA was extracted and processed by SMRT sequencing, which revealed a single contig of 6,500,439 bp in length with 320-fold sequence coverage (GenBank accession number: CP012679) (Li et al., 2016a). The result of SMRT sequencing revealed that the methylation status of PA1RG did not differ from that of PA1 (data unpublished). The circular genome map of PA1RG is shown in Figure 2A. Compared with the genome sequence of PA1, the PA1r (Red mutant) genome contains several large variations, such as inverted regions, 
A

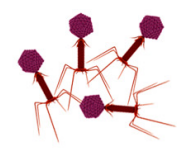

Phage PaP1

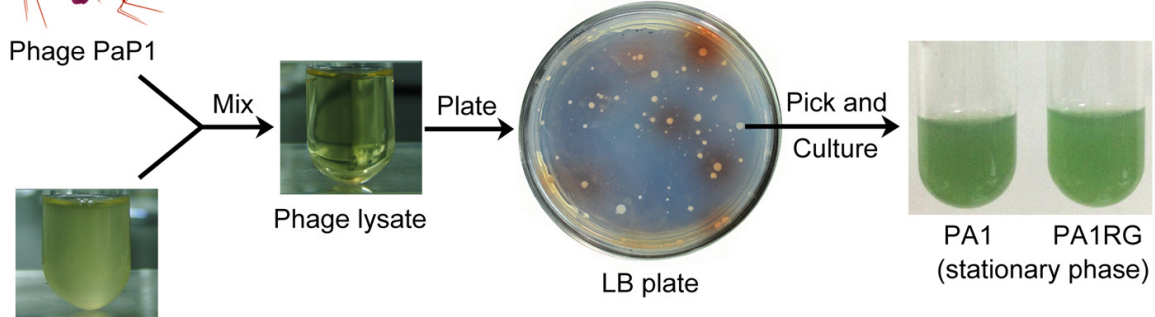

PA1 (log phase)

B

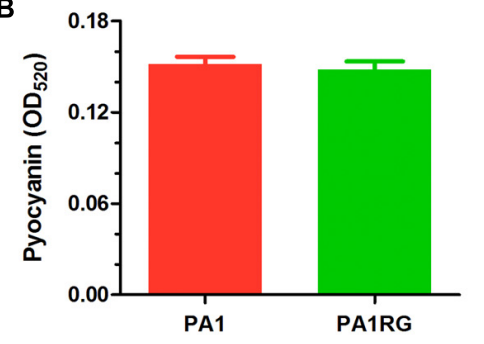

C

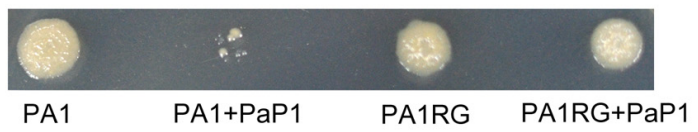

FIGURE 1 | Isolation of phage-resistant mutant PA1RG. (A) Diagram of PA1RG separation process. (B) Quantitation of pyocyanin production. Data were expressed as mean \pm standard deviation (SD) from three biological replicates. (C) Dot assay on agar plate showed that PA1RG was steady resistant to phage PaP1.

contig rearrangements, and a deletion with $219.6 \mathrm{~kb}$ in length (Figure 2B). By contrast, the PA1RG genome sequence exhibited no large variation compared to that of PA1 (Figure 2B).

\section{Genomic Variation Analyses Revealed the Mutation of Gene PA1S_08510}

Though no large variation was found in the mutant PA1RG genome, eight single nucleotide mutations were identified compared to the published PA1 genome sequence, including five insertions, two deletions, and one base transition (Figure 3A). Further analysis of the local sequence context of these mutated sites indicated that for the seven InDel (insertion/deletion) variations, each of the altered nucleotides was located within a continuous repeat of the same base. Taking number 2 mutation for example, the corresponding DNA sequence $5^{\prime}$-TCCCCCCG$3^{\prime}$ within PA1 was changed to $5^{\prime}$-TCCCCCCCG-3' after the insertion of the nucleotide cytosine within PA1RG genome. Among the eight variations, five were located in intergenic regions with no regulatory functions predicted, and three were in intragenic regions that matched the PA1 genes PA1S_13010, PA1S_28420, and PA1S_08510, respectively (Figure 3A). The detailed genetic variations identified in the PA1RG genome are summarized in Figure 3B. Both genes PA1S_13010 and PA1S_28420 were annotated as pseudo genes within the PA1 genome. Gene PA1S_08510 encodes a protein which is 417 amino acids in length, and the transition from cytosine to thymine in gene PA1S_08510 results in a stop codon and premature formation of a truncated product (198 amino acids in length). Thus, we focused our further study on the gene PA1S_08510.

\section{Gene PA1S_08510 Encodes a Protein Homologous to Wzy, an O-Antigen Polymerase}

BlastP alignment revealed that protein PA1S_08510 was highly conserved ( $>90 \%$ identity) among various $P$. aeruginosa strains, and almost all of the homologs were recorded as Wzy, an $O$-antigen polymerase encoded by the gene $w z y$. The interval spanning of 25-396 amino acid of PA1S_08510 constituted a conserved domain designated as glyco_rpt_poly (TIGR04370), which is suggested to participate in LPS O-antigen biosynthesis (Marchler-Bauer et al., 2017). Therefore, we proposed that PA1S_08510 could be named as PA1_Wzy.

PA1_Wzy exhibited 100\% identity with Wzy of P. aeruginosa strain PAK (serotype O6) (Figure 4A), and the functionality of PAK_Wzy (Y880_RS05480) has been genetically confirmed (Pan et al., 2016). By contrast, PA1_Wzy displayed limited sequence identity (35\%) with the active Wzy (PA3154) encoded by $P$. aeruginosa strain PAO1 (serotype O5) (Islam et al., 2010) (Figure 4A). Phylogenetic and alignment analysis showed that the primary sequences of $O$-antigen polymerase Wzy differ greatly among the $P$. aeruginosa strains that belong to different serotypes (Islam and Lam, 2014) (Figure 4B), and PA1_Wzy was classified into the O6 serotype based on sequence homology (Figure 4B). Despite the limited sequence conservation between PA1_Wzy and PAO1_Wzy, the two proteins adopt a similar transmembrane topology, especially for the presence of a large periplasmic loop at the C-terminal region (Figure 4C). 


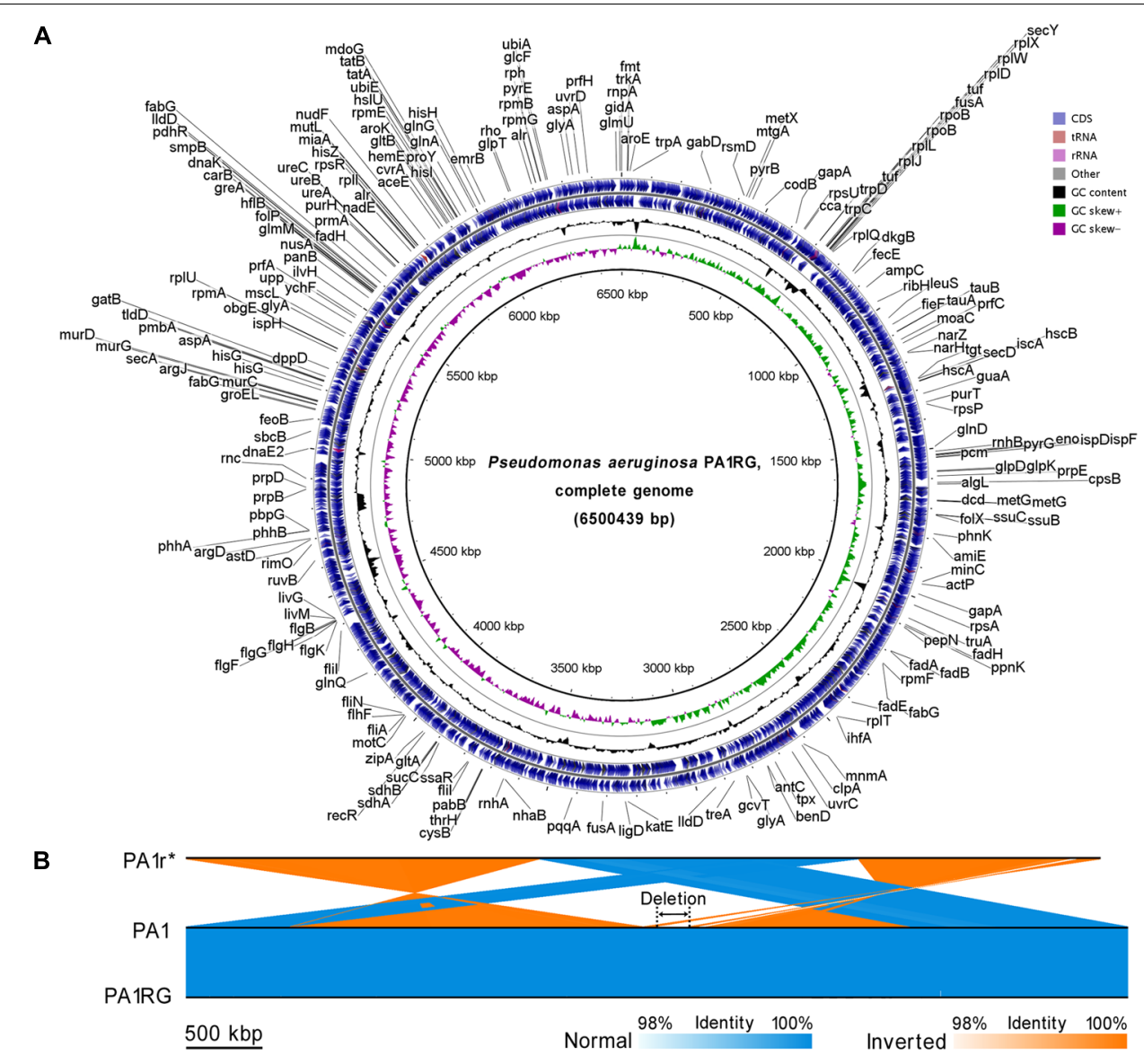

FIGURE 2 | Circular presentation and pairwise comparison of the PA1RG genome. (A) Diagram of the P. aeruginosa PA1RG genome. The names of 218 annotated genes (also shown in the GenBank file of the PA1RG genome) were indicated in black in the outermost region. The outermost ring depicts the genes on the plus strand, followed by rings depicting the genes on the minus strand, the GC content (black), and GC skew (green/purple). (B) Pairwise nucleotide sequence comparison of PA1r, PA1, and PA1RG. The length of the deletion region is $219.6 \mathrm{~kb}$. *In the GenBank file, the name of the Red mutant is PA1R, which is identical to PA1r.

\section{wzy Mutation Resulted in an LPS Defect in PA1RG}

Lipopolysaccharide is a complex and integral component of the bacterial cell envelope and typically consists of three structural domains: lipid A, core oligosaccharide, and the distal $O$-antigen (Lam et al., 2011). In the Wzy-dependent LPS biosynthetic pathway, the $\mathrm{O}$-antigen repeat unit is firstly polymerized via Wzy to form the long-chain polymer, followed by anchoring to the lipid core by the ligase, WaaL (Rocchetta et al., 1999). Bacteria lacking Wzy would produce known semi-rough LPS containing lipid A-core plus only one repeat unit on the cell surface (Lam et al., 2011).

To profile the LPS phenotype, the LPS of strain PA1 and PA1RG was extracted and subjected to SDS-PAGE analysis. Silver-stained SDS-PAGE gel showed that wild-type PA1 produced the normal LPS pattern, whereas mutant PA1RG contained the core oligosaccharide capped with one repeat unit, but failed to form the $\mathrm{O}$-antigen with high molecular weight (Figure 5A), demonstrating the semi-rough LPS phenotype of mutant PA1RG. To further validate the activity of PA1_Wzy, the wild-type wzy gene was transformed into mutant PA1RG. The LPS profile of the complementary strain PA1RG/wzy was then determined and displayed the same pattern to that of wild-type PA1 (Figure 5A). This finding revealed that PA1_Wzy provided in trans restored the long chain $O$-antigen biosynthesis in mutant PA1RG and functioned as an active $O$-antigen polymerase.

\section{Decreased Adsorption Confers PA1RG Resistance to Phage PaP1}

The initial and essential step of phage infection is the attachment of phage particles to the bacterial cell surface via receptor-ligand interactions. Adsorption inhibition caused by receptor mutation is usually identified in phage-resistant variants (Labrie et al., 2010). A previous study suggested that phage PaP1 recognizes the long chain $O$-antigen of PA1 LPS as its binding receptor (Le et al., 2013). To determine whether or not the wzy-mutationcaused LPS defect could inhibit the adsorption of PaP1 to PA1RG, an adsorption assay was performed. Compared with the ability of the attachment to the wild-type strain PA1, that of phage PaP1 attachment was significantly decreased to mutant 


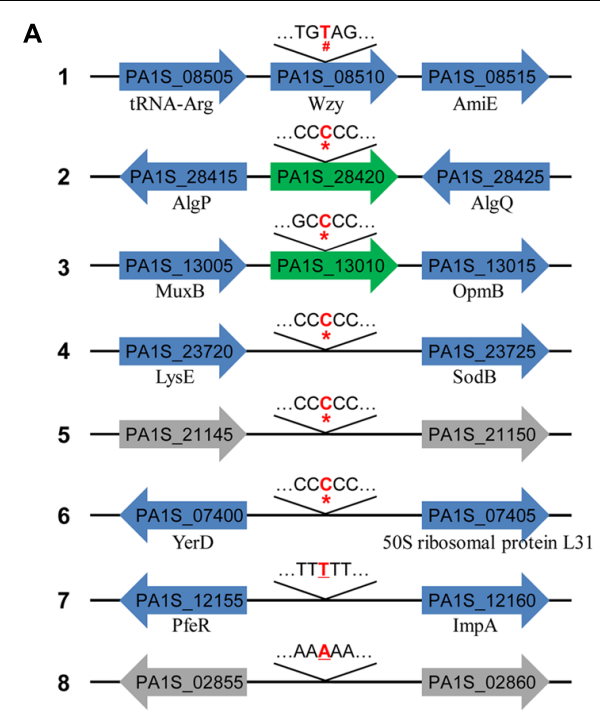

\begin{tabular}{|c|c|c|c|c|}
\hline No. & $\begin{array}{c}\text { Position } \\
\text { (bp, in } \\
\text { PA1) }\end{array}$ & $\begin{array}{c}\text { Type* }^{*} \\
\text { (PA1 } \rightarrow \\
\text { PA1RG) }\end{array}$ & Region & $\begin{array}{l}\text { Gene } \\
\text { locus } \\
\text { (in PA1) }\end{array}$ \\
\hline 1 & 1794539 & $\mathrm{C} \rightarrow \mathrm{T}$ & Intragenic & $\begin{array}{l}\text { PA1S } \\
08510\end{array}$ \\
\hline 2 & 6141784 & $\mathrm{I} \rightarrow \mathrm{C}$ & Intragenic & $\begin{array}{l}\text { PA1S } \\
28420\end{array}$ \\
\hline 3 & 2734990 & $\mathrm{I} \rightarrow \mathrm{C}$ & Intragenic & $\begin{array}{l}\text { PA1S } \\
13010\end{array}$ \\
\hline 4 & 5105452 & $1 \rightarrow C$ & Intergenic & \\
\hline 5 & 4536201 & $1 \rightarrow C$ & Intergenic & \\
\hline 6 & 1555125 & $1 \rightarrow C$ & Intergenic & \\
\hline 7 & 2545438 & $T \rightarrow I$ & Intergenic & \\
\hline 8 & 616184 & $\mathrm{~A} \rightarrow \mathrm{I}$ & Intergenic & \\
\hline
\end{tabular}

FIGURE 3 | Genetic variations identified within the PA1RG genome. (A) Diagram of mutations of the PA1RG genome sequence. Mutated nucleotides were labeled as red and bold letters, the symbol of \#, *, and underline indicates nucleotide substitution, insertion, and deletion, respectively. Green arrows represent pseudo genes, gray arrows represent genes encoding hypothetical protein, blue arrows represent functional genes and products are labeled underneath. Genes were not drawn to the scale. (B) Summary of genetic variations within the PA1RG genome. ${ }^{*}$ The symbol of/indicates absence of nucleotide in corresponding genomic site.

\section{A}

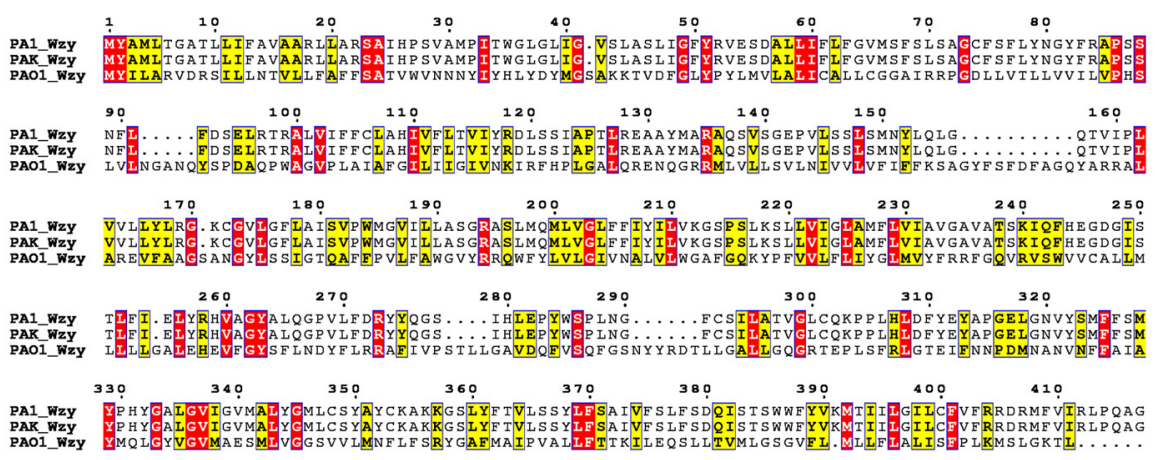

B

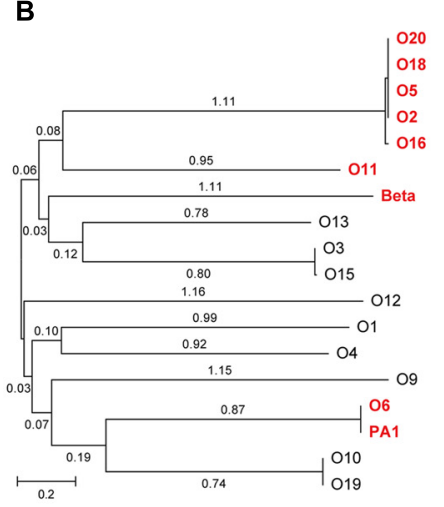

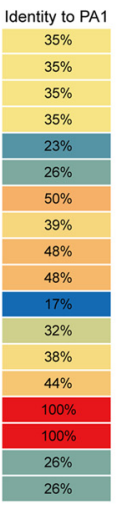

C
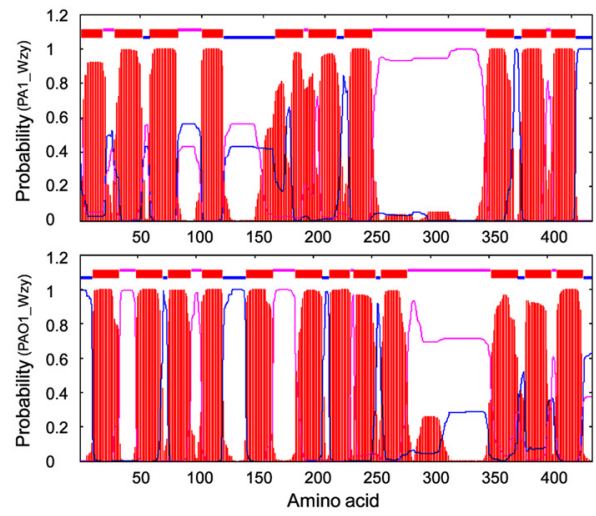

FIGURE 4 | In silico analyses of PA1_Wzy. (A) Sequence alignment of Wzy. Identical residues are shown as white letters boxed in red, similar residues are written with black characters boxed in yellow. (B) Phylogenetic analysis. Wzy homologs belonging to different $P$. aeruginosa serotypes were subjected to phylogenetic analysis. The serotypes labeled in red and bold letters indicate that Wzy activity has been experimentally determined in these serotypes. Beta represents phage D3-coding Wzy that mediates $\beta$-linkage of $O$-antigen repeat unit. (C) Transmembrane topology analysis. Red: transmembrane helices. Blue: cytoplasmic loops. Pink: periplasmic loops. 

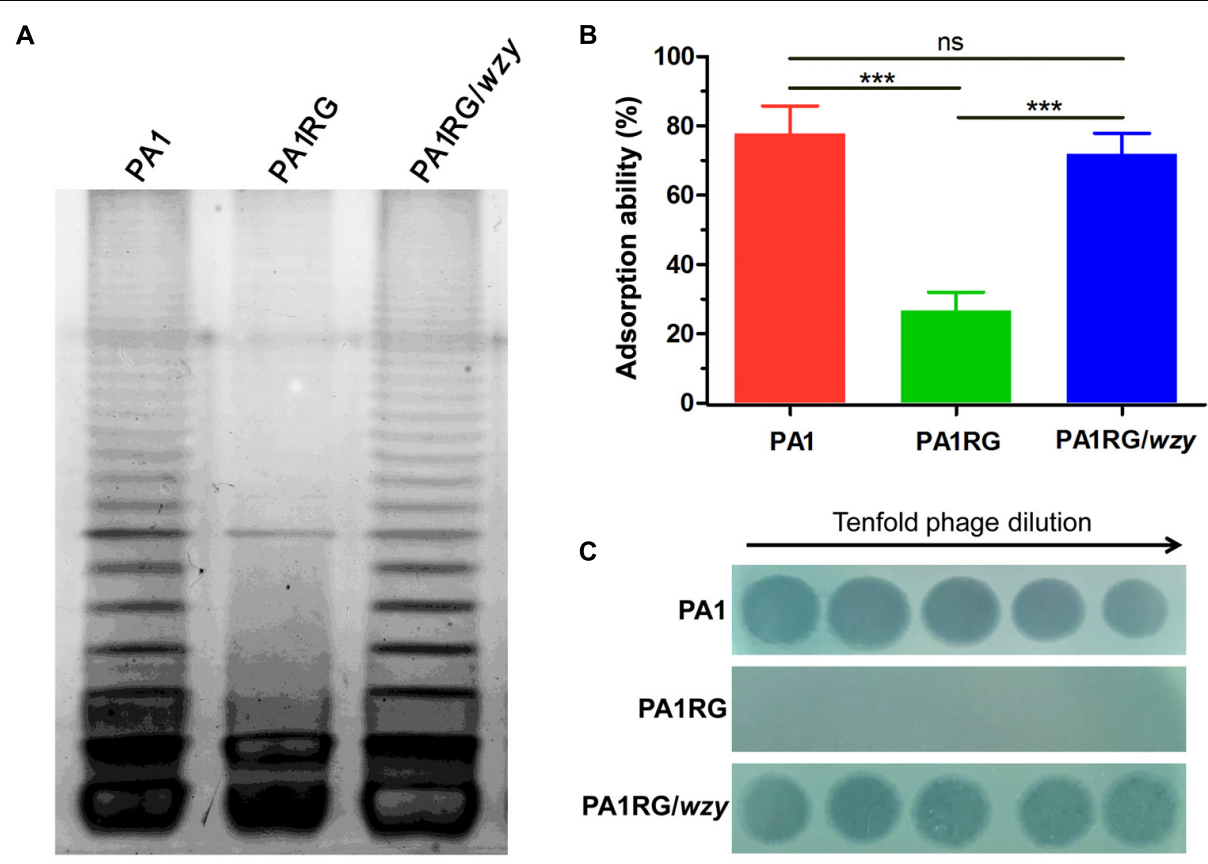

FIGURE 5 | LPS profiling, adsorption assay, and spot assay of PA1RG in comparison with PA1 and PA1RG/wzy. (A) LPS profiling. Mutant PA1RG produced LPS lacking long chain O-antigen. Complementary strain PA1RG/wzy displayed the same LPS pattern to that of wild-type PA1. (B) Adsorption assay. Decreased PaP1 attachment was observed for mutant PA1RG, which could be complemented by providing wzy in trans. Data were expressed as mean \pm SD from three biological replicates. ns, not significant. ${ }^{* *} p<0.001$. (C) Spot assay. Tenfold serial dilutions of PaP1 lysates were applied to the lawns of indicated strains. Phage-resistant PA1RG restored PaP1 susceptibility when bearing the intact gene wzy.

PA1RG, but restored to the complementary strain PA1RG/wzy (Figure 5B). These results confirmed that $w z y$-mutation-caused LPS truncation reduced the adsorption of phage PaP1 to PA1RG.

To further define the role of PA1_Wzy in regulating PA1RG resistance to phage $\mathrm{PaP} 1$, a spot assay was performed for the complementary strain PA1RG/wzy. Mutant PA1RG restored the susceptibility to phage $\mathrm{PaP} 1$ when possessing the intact gene $w z y$ in trans (Figure 5C), demonstrating that $w z y$ mutation caused $\mathrm{PA} 1 \mathrm{RG}$ resistance to $\mathrm{PaP} 1$ predation.

\section{Reduced Biofilm Formation for Phage-Resistant PA1RG}

As described in various phage-host interactions, phage predation could pose multifaceted effects on bacterial biology, such as growth, motility, and virulence (Hosseinidoust et al., 2013b; Feiner et al., 2015; De Smet et al., 2017). To assess the potential effects of phage resistance on PA1RG biology, we firstly profiled the growth characteristics of PA1 and PA1RG. The growth rate was not significantly different for the two strains under the tested conditions (Figure 6A). However, the ability of mutant PA1RG to produce biofilm was significantly decreased compared with that of the parental strain PA1, and this deficiency could be offset by providing the wild-type gene $w z y$ in trans (Figure 6B). CLSM analysis of biofilm formation further demonstrated that mutant PA1RG produced less and thinner biofilm compared to both strain PA1 and PA1RG/wzy (Figure 7), indicating a trade-off between phage resistance and biofilm-forming ability.

\section{DISCUSSION}

Phages are the most diverse and abundant genetic entities on earth with an estimated number of $10^{31}$ in total (Sharma et al., 2017). The highly broad distribution of phages, particularly for lytic phages, poses a major threat to the survival of their bacterial hosts (Samson et al., 2013). As a result, bacteria have evolved various defense systems to evade and survival phage predation. Known mechanisms include adsorption inhibition, R-M system, CRISPR/Cas system, and Abi system, which function and target every possible step of phage infection (Labrie et al., 2010). Attachment of phage to host cell surface, the initial and essential step to complete phage infection cycle, is usually impaired among phage-resistant bacteria (Labrie et al., 2010). In this study, we elucidated the molecular basis for $P$. aeruginosa PA1RG resistance to phage PaP1. The transition mutation within gene $w z y$ resulted in the premature formation of $\mathrm{O}$-antigen polymerase, which is involved in long-chain $\mathrm{O}$-antigen biosynthesis related to $\mathrm{PaP} 1$ recognition and binding. Combined with the previously described resistance mechanism for Red mutant PA1r, which lost a large DNA segment $(219.6 \mathrm{~kb})$ containing the key gene galU responsible for core oligosaccharide biosynthesis (Le et al., 2014), these results enhanced our understanding of the multiple mechanisms that $P$. aeruginosa used to resist phage predation.

The receptor on the bacterial cell surface is responsible for phage attachment and mirrors the diversity of phage types (Rakhuba et al., 2010). To date, various receptors recognized 
A

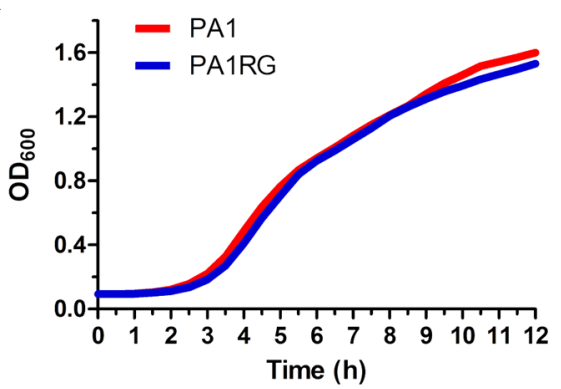

B

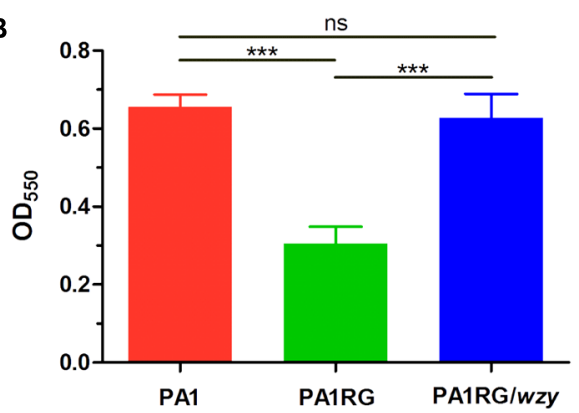

FIGURE 6 | Growth and biofilm assay. (A) Growth curves of PA1 and PA1RG that monitored every 30 min. Data represent the average of three biological replicates. (B) Microtiter dish biofilm formation assay. Data were expressed as mean \pm SD from three biological replicates. ns, not significant. $* * * p<0.001$.

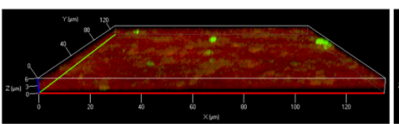

PA1

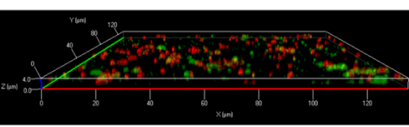

PA1RG

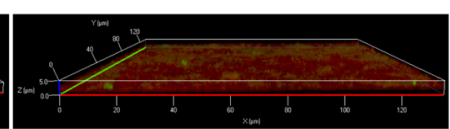

PA1RG/wzy

FIGURE 7 | CLSM analysis of biofilm formation. Bacterial cells in biofilm were labeled with the red fluorescent nucleic acid stain SYTO 61, and extracellular polysaccharides were labeled with the green fluorescent stain FITC-ConA. Mutant PA1RG produced thinner biofilm compared to PA1 and PA1RG/wzy.

by $P$. aeruginosa phages have been identified. These receptors include type IV pili (Kim et al., 2012), O-antigen (Le et al., 2014), common polysaccharide antigen (Rivera et al., 1992), core oligosaccharide (Temple et al., 1986), and outer membrane protein (Chan et al., 2016b). Interestingly, phages PaP1, K8, and $\mathrm{K} 5$, all belonging to the genus of Pakpunaviruses and using the $O$-antigen as receptor, infect $P$. aeruginosa strain PA1 and PAK, respectively (Lu et al., 2013; Li et al., 2016c; Pan et al., 2016). Phage-resistant variants possessing mutation in gene wzy were selected from both PA1 and PAK upon corresponding phage predation (Li et al., 2016c; Pan et al., 2016), suggesting that $w z y$ mutation may represent a preferred resistance mechanism for phages of Pakpunaviruses.

The phenotype of phage-resistant Green mutant PA1RG producing decreased biofilm, and the previously identified Red mutant PA1r displaying a growth defect, illustrated the diverse and distinct effects of phage infection on bacteria biology. The similar trade-off between phage resistance and bacteria functionality has been described in various phage$P$. aeruginosa interactions, which mainly influenced $P$. aeruginosa biofilm formation, virulence, and/or antibiotic resistance (De Smet et al., 2017). The type IV pili dependent phage D3112 selected for $P$. aeruginosa mutants that showed decreased surface adherence and biofilm formation, but increased antibiotic sensitivity (Hosseinidoust et al., 2013a). The use of LPS as receptor by phage E79 resulted in LPS alterations of phageresistant variants, which in turn lead to decreased swarming and increased biofilm formation (Hosseinidoust et al., 2013a). Serotype conversion of $P$. aeruginosa PAO1 from O5 to O16 as mediated by temperate phage D3 enables bacterial resistance to superinfection by related phages, and also contributes to enhanced adherence and resistance to animal immune systems
(Newton et al., 2001; Taylor et al., 2013). Reversion of antibiotic susceptibility was conferred by lytic phage OMKO1 (Chan et al., 2016b), which recognizes and binds to the outer membrane porin $\mathrm{M}$ involved in multidrug efflux. OMKO1-resistant mutant exhibited reduced antibiotic resistance owing to the decreased expression of porin M (Chan et al., 2016b). Despite the high conservation between phage $\mathrm{PaP} 1$ and $\mathrm{K} 8$, mutants against each infecting phage displayed reduced and increased biofilm, respectively (Pan et al., 2016), indicating the conflicting effects of phage predation. Based on the known cases, the diverse effects (either positive or negative) posed by $P$. aeruginosa phages exhibit features of complexity and unpredictability and might be phage and/or host-specific (Chaturongakul and Ounjai, 2014; De Smet et al., 2017).

As natural enemies for bacteria, phages were immediately used to treat bacterial infections upon their discovery approximately 100 years ago (Cisek et al., 2017), and interests in their potential as antimicrobials increasing mainly due to the global threat of antibiotic resistance (Nobrega et al., 2015). To efficiently facilitate the application of phage therapy, a comprehensive understanding of phage-host interactions is required. In this study, we revealed that $\mathrm{PaP} 1$ infection resulted in mutant PA1RG with decreased biofilm formation. Biofilm represents an important characteristic that greatly benefits $P$. aeruginosa with defense against antibiotic treatment and prolonged survival especially during chronic lung infection (Stover et al., 2000). The trade-off between phage-resistance and fitness cost may be a potential advantage for phage therapy to treat bacterial infections. Furthermore, a dual therapy with combined phage and antibiotic has displayed increased antibacterial effect compared with the sole use of each agent in several studies (Viertel et al., 2014). For example, methicillin-resistant Staphylococcus aureus was 
efficiently eliminated from diabetic foot infections using phage MR-10 and linezolid (Chhibber et al., 2013), the eradication of Klebsiella pneumonia biofilm was increased by the combination of ciprofloxacin and phage KPO1K2 producing the depolymerase (Verma et al., 2010), and limited evolution of bacterial resistance to kanamycin was conferred by phage SBW25Ф2 predation in P. fluorescens (Zhang and Buckling, 2012). With the impressive advantages of synergistic antibacterial effect and less frequently evolved resistance (Chan et al., 2016a), the application of phage and antibiotic in combination represents a promising strategy to control bacterial infections, especially for those caused by biofilm-forming and multi-drug resistant bacteria (Tillotson and Theriault, 2013; Parasion et al., 2014).

\section{REFERENCES}

Bondy-Denomy, J., Qian, J., Westra, E. R., Buckling, A., Guttman, D. S., Davidson, A. R., et al. (2016). Prophages mediate defense against phage infection through diverse mechanisms. ISME J. 10, 2854-2866. doi: 10.1038/ismej.2016.79

Chan, B. K., Brown, K., Kortright, K. E., Mao, S., and Turner, P. E. (2016a). Extending the lifetime of antibiotics: how can phage therapy help? Future Microbiol. 11, 1105-1107. doi: 10.2217/fmb-2016-0133

Chan, B. K., Sistrom, M., Wertz, J. E., Kortright, K. E., Narayan, D., and Turner, P. E. (2016b). Phage selection restores antibiotic sensitivity in MDR Pseudomonas aeruginosa. Sci. Rep. 6:26717. doi: 10.1038/srep26717

Chaturongakul, S., and Ounjai, P. (2014). Phage-host interplay: examples from tailed phages and Gram-negative bacterial pathogens. Front. Microbiol. 5:442. doi: 10.3389/fmicb.2014.00442

Chhibber, S., Kaur, T., and Kaur, S. (2013). Co-therapy using lytic bacteriophage and linezolid: effective treatment in eliminating methicillin resistant Staphylococcus aureus (MRSA) from diabetic foot infections. PLOS One 8:e56022. doi: 10.1371/journal.pone.0056022

Chin, C. S., Alexander, D. H., Marks, P., Klammer, A. A., Drake, J., Heiner, C., et al. (2013). Nonhybrid, finished microbial genome assemblies from long-read SMRT sequencing data. Nat. Methods 10, 563-569. doi: 10.1038/nmeth.2474

Cisek, A. A., Dabrowska, I., Gregorczyk, K. P., and Wyzewski, Z. (2017). Phage therapy in bacterial infections treatment: one hundred years after the discovery of bacteriophages. Curr. Microbiol. 74, 277-283. doi: 10.1007/s00284-0161166-x

De Smet, J., Hendrix, H., Blasdel, B. G., Danis-Wlodarczyk, K., and Lavigne, R. (2017). Pseudomonas predators: understanding and exploiting phage-host interactions. Nat. Rev. Microbiol. 15, 517-530. doi: 10.1038/nrmicro.2017.61

Essar, D. W., Eberly, L., Hadero, A., and Crawford, I. P. (1990). Identification and characterization of genes for a second anthranilate synthase in Pseudomonas aeruginosa: interchangeability of the two anthranilate synthases and evolutionary implications. J. Bacteriol. 172, 884-900. doi: 10.1128/jb.172.2. 884-900.1990

Feiner, R., Argov, T., Rabinovich, L., Sigal, N., Borovok, I., and Herskovits, A. A. (2015). A new perspective on lysogeny: prophages as active regulatory switches of bacteria. Nat. Rev. Microbiol. 13, 641-650. doi: 10.1038/nrmicro3527

Fomsgaard, A., Freudenberg, M. A., and Galanos, C. (1990). Modification of the silver staining technique to detect lipopolysaccharide in polyacrylamide gels. J. Clin. Microbiol. 28, 2627-2631.

Hosseinidoust, Z., Tufenkji, N., and van de Ven, T. G. (2013a). Formation of biofilms under phage predation: considerations concerning a biofilm increase. Biofouling 29, 457-468. doi: 10.1080/08927014.2013.779370

Hosseinidoust, Z., van de Ven, T. G. M., and Tufenkji, N. (2013b). Evolution of Pseudomonas aeruginosa virulence as a result of phage predation. Appl. Environ. Microbiol. 79, 6110-6116. doi: 10.1128/Aem.01421-13

Islam, S. T., and Lam, J. S. (2014). Synthesis of bacterial polysaccharides via the Wzx/Wzy-dependent pathway. Can. J. Microbiol. 60, 697-716. doi: 10.1139/ cjm-2014-0595

Islam, S. T., Taylor, V. L., Qi, M., and Lam, J. S. (2010). Membrane topology mapping of the $\mathrm{O}$-antigen flippase (Wzx), polymerase (Wzy), and ligase (WaaL)

\section{AUTHOR CONTRIBUTIONS}

GL, MS, YY, and SL performed the experiments. GL, ML, and SL analyzed the data. SL, JW, YZ, and YT contributed reagents and materials. FH, SL, and GL designed the experiments and wrote the paper.

\section{FUNDING}

This work was supported by the National Natural Science Foundation of China (Grant No. 31570173 and 3140 0163).

from Pseudomonas aeruginosa PAO1 reveals novel domain architectures. $m$ Bio 1:e189-10. doi: 10.1128/mBio.00189-10

Kim, S., Rahman, M., Seol, S. Y., Yoon, S. S., and Kim, J. (2012). Pseudomonas aeruginosa bacteriophage PA1O requires type IV pili for infection and shows broad bactericidal and biofilm removal activities. Appl. Environ. Microbiol. 78, 6380-6385. doi: 10.1128/AEM.00648-12

Krogh, A., Larsson, B., von Heijne, G., and Sonnhammer, E. L. (2001). Predicting transmembrane protein topology with a hidden Markov model: application to complete genomes. J. Mol. Biol. 305, 567-580. doi: 10.1006/jmbi.2000. 4315

Labrie, S. J., and Moineau, S. (2007). Abortive infection mechanisms and prophage sequences significantly influence the genetic makeup of emerging lytic Lactococcal phages. J. Bacteriol. 189, 1482-1487. doi: 10.1128/JB.01 111-06

Labrie, S. J., Samson, J. E., and Moineau, S. (2010). Bacteriophage resistance mechanisms. Nat. Rev. Microbiol. 8, 317-327. doi: 10.1038/nrmicro2315

Lam, J. S., Taylor, V. L., Islam, S. T., Hao, Y., and Kocincova, D. (2011). Genetic and functional diversity of Pseudomonas aeruginosa lipopolysaccharide. Front. Microbiol. 2:118. doi: 10.3389/fmicb.2011.00118

Larkin, M. A., Blackshields, G., Brown, N. P., Chenna, R., McGettigan, P. A., McWilliam, H., et al. (2007). Clustal W and clustal X version 2.0. Bioinformatics 23, 2947-2948. doi: 10.1093/bioinformatics/btm404

Le, S., He, X. S., Tan, Y. L., Huang, G. T., Zhang, L., Lux, R., et al. (2013). Mapping the tail fiber as the receptor binding protein responsible for differential host specificity of Pseudomonas aeruginosa bacteriophages PaP1 and JG004. PLOS One 8:e68562. doi: 10.1371/journal.pone.0068562

Le, S., Yao, X., Lu, S., Tan, Y., Rao, X., Li, M., et al. (2014). Chromosomal DNA deletion confers phage resistance to Pseudomonas aeruginosa. Sci. Rep. 4:4738. doi: 10.1038/srep04738

Li, G., Lu, S., Shen, M., Le, S., Shen, W., Tan, Y., et al. (2017). Characterization and interstrain transfer of prophage pp3 of Pseudomonas aeruginosa. PLOS One 12:e0174429. doi: 10.1371/journal.pone.0174429

Li, G., Lu, S., Shen, M., Le, S., Tan, Y., Li, M., et al. (2016a). Complete genome sequence of Pseudomonas aeruginosa phage-resistant variant PA1RG. Genome Announc. 4:e1761-15. doi: 10.1128/genomeA.01761-15

Li, G., Shen, M., Le, S., Tan, Y., Li, M., Zhao, X., et al. (2016b). Genomic analyses of multidrug resistant Pseudomonas aeruginosa PA1 resequenced by single-molecule real-time sequencing. Biosci. Rep. 36:e000418. doi: 10.1042/ BSR20160282

Li, L., Pan, X., Cui, X., Sun, Q., Yang, X., and Yang, H. (2016c). Characterization of Pseudomonas aeruginosa phage K5 genome and identification of its receptor related genes. J. Basic Microbiol. 56, 1344-1353. doi: 10.1002/jobm.201600116

Lu, S., Le, S., Li, G., Shen, M., Tan, Y., Zhao, X., et al. (2015). Complete genome sequence of Pseudomonas aeruginosa PA1, isolated from a patient with a respiratory tract infection. Genome Announc. 3:e1453-15. doi: 10.1128/ genomeA.01453-15

Lu, S., Le, S., Tan, Y., Zhu, J., Li, M., Rao, X., et al. (2013). Genomic and proteomic analyses of the terminally redundant genome of the Pseudomonas aeruginosa phage PaP1: establishment of genus PaP1-like phages. PLOS One 8:e62933. doi: 10.1371/journal.pone.0062933 
Marchler-Bauer, A., Bo, Y., Han, L., He, J., Lanczycki, C. J., Lu, S., et al. (2017). CDD/SPARCLE: functional classification of proteins via subfamily domain architectures. Nucleic Acids Res. 45, D200-D203. doi: 10.1093/nar/gkw1129

Mathee, K., Narasimhan, G., Valdes, C., Qiu, X., Matewish, J. M., Koehrsen, M., et al. (2008). Dynamics of Pseudomonas aeruginosa genome evolution. Proc. Natl. Acad. Sci. U.S.A. 105, 3100-3105. doi: 10.1073/pnas.0711982105

Michel, A., Clermont, O., Denamur, E., and Tenaillon, O. (2010). Bacteriophage PhiX174's ecological niche and the flexibility of its Escherichia coli lipopolysaccharide receptor. Appl. Environ. Microbiol. 76, 7310-7313. doi: 10.1128/AEM.02721-09

Newton, G. J., Daniels, C., Burrows, L. L., Kropinski, A. M., Clarke, A. J., and Lam, J. S. (2001). Three-component-mediated serotype conversion in Pseudomonas aeruginosa by bacteriophage D3. Mol. Microbiol. 39, 1237-1247. doi: 10.1111/j. 1365-2958.2001.02311.x

Nilsson, A. S. (2014). Phage therapy-constraints and possibilities. Ups. J. Med. Sci. 119, 192-198. doi: 10.3109/03009734.2014.902878

Nobrega, F. L., Costa, A. R., Kluskens, L. D., and Azeredo, J. (2015). Revisiting phage therapy: new applications for old resources. Trends Microbiol. 23, 185191. doi: 10.1016/j.tim.2015.01.006

O’Toole, G. A. (2011). Microtiter dish biofilm formation assay. J. Vis. Exp. 47:2437. doi: $10.3791 / 2437$

Pan, X. W., Cui, X. L., Zhang, F. J., He, Y., Li, L. Y., and Yang, H. J. (2016). Genetic evidence for o-specific antigen as receptor of Pseudomonas aeruginosa phage K8 and its genomic analysis. Front. Microbiol. 7:252. doi: 10.3389/Fmicb.2016. 00252

Parasion, S., Kwiatek, M., Gryko, R., Mizak, L., and Malm, A. (2014). Bacteriophages as an alternative strategy for fighting biofilm development. Pol. J. Microbiol. 63, 137-145.

Pepin, K. M., Domsic, J., and McKenna, R. (2008). Genomic evolution in a virus under specific selection for host recognition. Infect. Genet. Evol. 8, 825-834. doi: 10.1016/j.meegid.2008.08.008

Rakhuba, D. V., Kolomiets, E. I., Dey, E. S., and Novik, G. I. (2010). Bacteriophage receptors, mechanisms of phage adsorption and penetration into host cell. Pol. J. Microbiol. 59, 145-155.

Rivera, M., Chivers, T. R., Lam, J. S., and McGroarty, E. J. (1992). Common antigen lipopolysaccharide from Pseudomonas aeruginosa AK1401 as a receptor for bacteriophage A7. J. Bacteriol. 174, 2407-2411. doi: 10.1128/jb.174.7.24072411.1992

Robert, X., and Gouet, P. (2014). Deciphering key features in protein structures with the new ENDscript server. Nucleic Acids Res. 42, W320-W324. doi: 10. 1093/nar/gku316

Rocchetta, H. L., Burrows, L. L., and Lam, J. S. (1999). Genetics of O-antigen biosynthesis in Pseudomonas aeruginosa. Microbiol. Mol. Biol. Rev. 63, 523-553.

Saitou, N., and Nei, M. (1987). The neighbor-joining method: a new method for reconstructing phylogenetic trees. Mol. Biol. Evol. 4, 406-425.

Samson, J. E., Magadan, A. H., Sabri, M., and Moineau, S. (2013). Revenge of the phages: defeating bacterial defences. Nat. Rev. Microbiol. 11, 675-687. doi: 10.1038/nrmicro3096

Sharma, S., Chatterjee, S., Datta, S., Prasad, R., Dubey, D., Prasad, R. K., et al. (2017). Bacteriophages and its applications: an overview. Folia Microbiol. 62, 17-55. doi: 10.1007/s12223-016-0471-X

Stover, C. K., Pham, X. Q., Erwin, A. L., Mizoguchi, S. D., Warrener, P., Hickey, M. J., et al. (2000). Complete genome sequence of Pseudomonas aeruginosa PAO1, an opportunistic pathogen. Nature 406, 959-964. doi: 10.1038/35023079
Takenaka, S., Iwaku, M., and Hoshino, E. (2001). Artificial Pseudomonas aeruginosa biofilms and confocal laser scanning microscopic analysis. J. Infect. Chemother. 7, 87-93. doi: 10.1007/s1015610070087

Tamura, K., Stecher, G., Peterson, D., Filipski, A., and Kumar, S. (2013). MEGA6: molecular evolutionary genetics analysis version 6.0. Mol. Biol. Evol. 30, 27252729. doi: 10.1093/molbev/mst197

Taylor, V. L., Udaskin, M. L., Islam, S. T., and Lam, J. S. (2013). The D3 bacteriophage alpha-polymerase inhibitor (Iap) peptide disrupts o-antigen biosynthesis through mimicry of the chain length regulator Wzz in Pseudomonas aeruginosa. J. Bacteriol. 195, 4735-4741. doi: 10.1128/Jb.00903-13

Temple, G. S., Ayling, P. D., and Wilkinson, S. G. (1986). Isolation and characterization of a lipopolysaccharide-specific bacteriophage of Pseudomonas aeruginosa. Microbios 45, 81-91.

Tillotson, G. S., and Theriault, N. (2013). New and alternative approaches to tackling antibiotic resistance. F1000Prime Rep. 5:51. doi: 10.12703/P5-51

Turner, K. H., Wessel, A. K., Palmer, G. C., Murray, J. L., and Whiteley, M. (2015). Essential genome of Pseudomonas aeruginosa in cystic fibrosis sputum. Proc. Natl. Acad. Sci. U.S.A. 112, 4110-4115. doi: 10.1073/pnas.1419677112

Valot, B., Guyeux, C., Rolland, J. Y., Mazouzi, K., Bertrand, X., and Hocquet, D. (2015). What it takes to be a Pseudomonas aeruginosa? the core genome of the opportunistic pathogen updated. PLOS One 10:e0126468. doi: 10.1371/journal. pone. 0126468

Verma, V., Harjai, K., and Chhibber, S. (2010). Structural changes induced by a lytic bacteriophage make ciprofloxacin effective against older biofilm of Klebsiella pneumoniae. Biofouling 26, 729-737. doi: 10.1080/08927014.2010.51 1196

Viertel, T. M., Ritter, K., and Horz, H. P. (2014). Viruses versus bacteria-novel approaches to phage therapy as a tool against multidrug-resistant pathogens. J. Antimicrob. Chemother. 69, 2326-2336. doi: 10.1093/jac/dku173

West, S. E., Schweizer, H. P., Dall, C., Sample, A. K., and Runyen-Janecky, L. J. (1994). Construction of improved Escherichia-Pseudomonas shuttle vectors derived from pUC18/19 and sequence of the region required for their replication in Pseudomonas aeruginosa. Gene 148, 81-86. doi: 10.1016/03781119(94)90237-2

Yi, W., Liu, X. W., Li, Y. H., Li, J. J., Xia, C. F., Zhou, G. Y., et al. (2009). Remodeling bacterial polysaccharides by metabolic pathway engineering. Proc. Natl. Acad. Sci. U.S.A. 106, 4207-4212. doi: 10.1073/pnas.0812432106

Zhang, Q. G., and Buckling, A. (2012). Phages limit the evolution of bacterial antibiotic resistance in experimental microcosms. Evol. Appl. 5, 575-582. doi: $10.1111 /$ j.1752-4571.2011.00236.x

Conflict of Interest Statement: The authors declare that the research was conducted in the absence of any commercial or financial relationships that could be construed as a potential conflict of interest.

The reviewer KD-W and handling Editor declared their shared affiliation.

Copyright (c) 2018 Li, Shen, Yang, Le, Li, Wang, Zhao, Tan, Hu and Lu. This is an open-access article distributed under the terms of the Creative Commons Attribution License (CC BY). The use, distribution or reproduction in other forums is permitted, provided the original author(s) and the copyright owner are credited and that the original publication in this journal is cited, in accordance with accepted academic practice. No use, distribution or reproduction is permitted which does not comply with these terms. 